\title{
THE EVOLUTION TO STEADY NONLINEAR PULSATION IN STELLAR MODELS
}

\author{
G. Kovács and J. R. Buchler \\ University of Florida
}

\section{ABSTRACT}

The numerically generated dynamical evolution of an RR Lyrae model from different initial conditions is subjected to a timedependent Pourier analysis, which yields the temporal behavior of the amplitudes and phases of the few longlived transient modes in addition to the ultimate winner. It is shown that the amplitude equation formalism of Buchler and Goupil gives an almost perfect fit to the observed transient behavior of the amplitudes and phases of the excited modes. Prospects and applications are discussed.

In the last few years mathematical developments in the study of nonlinear dynamics have made possible a novel attack on many nonlinear stellar pulsators. In the context of pulsating stars these mathematical techniques are concerned with the reduction of the partial differential equations of hydrodynamics and heat-flow to a small set of differential equations governing the temporal behavior of the amplitudes and phases of just a few modes. Buchler and Goupil (1984; also Buchler 1985) used an asymptotic perturbation approach, specially tailored to the classical radial pulsators, whereas in the context of fluid dynamics, Coullet and Spiegel (1984; also Spiegel 1985) have followed a different approach, which ultimately, however, leads to the same equations. One of the advantages of these formalisms is that the solution of the partial differential system appears ultimately in terms of the same quantities that a Fourier analysis of the stellar observations yields. An accurate and direct comparison with observation becomes thus feasible (Buchler and Kovacs $1986 a, b)$. Not only is the application of this formalism enormously faster in terms of computer time (e. g. Klapp, Goupil and Buchler 1985), but more importantly, it yields fresh physical insight into the pulsation mechanism, and this is bound eventually to lead to a better understanding of the structure and evolution of of the pulsating stars.

Here we shall demonstrate that the numerically generated evolution of a realistic RR Lyrae model from some initial conditions to its final steady state can readily be accomodated and described within our formalism. The stellar model, which we shall describe has a mass of 0.65 and luminosity of 60 , both in solar units, an effective temperature of $7000 \mathrm{~K}$ and $\mathrm{a}$ composition of $(\mathrm{X}=0.700, \mathrm{Z}=0.001)$. The numerical hydrodynamic integration of the model has been performed with Stellingwerf's (1974) code (with a rigid inner boundary) with 60 mass-shells, 20 equal ones up to 20,000 $K$ and the rest reaching up to $\sim 2$ million degrees. The viscosity parameter are $C_{0}=4.0$ and $\alpha=0.01$. The 1 st run has been initiated with a velocity profile corresponding to the linear fundamental eigenvector with a $10 \%$ admixture of the 1 st overtone and the 2 nd run with a $2 \%$ admixture, both with a $10 \mathrm{~km} / \mathrm{s}$ surface velocity. 
The variation of the outer radius is first subjected to a timedependent Fourier analysis (Kovacs, Buchler and Davis 1986), which consists of a Maximum Entropy Method determination of the 8 lowest frequencies $\left\{f_{k}\right\}$, performed over some fraction of the total timebase.

$\begin{array}{lll}\mathrm{f}_{1}=1.848 & \sigma_{0} & =1.8478 \\ \mathrm{f}_{2}=2.478 & \sigma_{1} & =2.4787 \\ \mathrm{f}_{3}=3.109 & 2 \sigma_{1}-\sigma_{0} & =3.1096 \\ \mathrm{f}_{4}=3.697 & 2 \sigma_{0} & =3.6956 \\ \mathrm{f}_{5}=4.326 & \sigma_{0}+\sigma_{1} & =4.3265 \\ \mathrm{f}_{6}=0.631 & \sigma_{1}-\sigma_{0} & =0.6309 \\ \mathrm{f}_{7}=1.254 & 2 \sigma_{0}-\sigma_{1} & =1.2169 \\ \mathrm{f}_{8}=4.957 & 2 \sigma_{1} & =4.9574\end{array}$$$
\begin{array}{ll}
v_{0}=1.8488 & k_{0}=0.0078 \\
v_{1}=2.4814 & k_{1}=0.0547 \\
v_{2}=3.1094 & k_{2}=-0.0134 \\
v_{3}=3.8220 & k_{3}=-0.2884
\end{array}
$$

(frequencies in cycles/day, growth-rates in day $^{-1}$,

The second column shows the interpretation of these frequencies in terms of two basic ones, $\sigma_{0}$ and $\sigma_{1}$, and their combinations. For reference are also shown the linear nonadiabatic frequencies $\left\{v_{k}\right\}$ and growth-rates $\left\{\kappa_{k}\right\}$. It is clear that $\sigma_{0}$ and $\sigma_{1}$ correspond to the fundamental and first overtones. The other modes, if present, are so short-lived that they do not appreciably show up.

A large number of fits of the hydro-output is next performed with a Fourier sum over successive time-intervals, in each of which the amplitudes and phases are held constant ('instantaneous' quantities):

$$
R(t)=A_{0}(t)+\sum_{k=1}^{K} A_{k}(t) \sin \left\{2 \pi f_{k}\left(t-t_{0}\right)+\phi_{k}\right\}
$$

where the $\mathrm{E}_{\mathrm{k}}$ denote the combinations in column 2 above, supplemented by additional third order ones. The result is an accurate determination of the temporal behavior of the amplitudes and phases.

The next step consists in ascertaining that the behavior of these amplitudes and phases can well be described by normal form amplitude equations. We shall attempt a description in terms of two nonresonant modes, the fundamental and the first overtone. The relevant equations for the (real) amplitudes and phases (e. g. Buchler 1985) are given by

$$
\begin{aligned}
& \frac{d A}{d t}=K_{0} A+\operatorname{Re} Q_{0} A^{3}+\operatorname{Re} T_{0} A B^{2} \\
& \frac{d B}{d t}=K_{1} B+\operatorname{Re} Q_{1} B^{3}+\operatorname{Re} T_{1} B A^{2} \\
& \frac{d \phi_{0}}{d t}=\omega_{0}+\operatorname{Im} Q_{0} A^{2}+\operatorname{Im} T_{0} B^{2} \\
& \frac{d \phi}{d t} 1=\omega_{1}+\operatorname{Im} Q_{1} B^{2}+\operatorname{Im} T_{1} A^{2}
\end{aligned}
$$

where $A$ and $B$ denote the amplitudes of the two modes and the $\phi_{y}$ the corresponding phases. Higher powers in the amplitudes have been neglected. 
The surface radius variation, to lowest order, is

$\frac{\delta R}{R}=\left\{\frac{1}{2} A(t) e^{i \omega_{0} t+i \phi_{0}}+\frac{1}{2} B(t) e^{i \omega_{1} t+i \phi_{1} t}+c \cdot c \cdot\right\}+\cdots$

The normal form equations for the amplitudes and phases are decoupled. The integral curves of the phase equations are linear in the coefficients and the $f i t$ is trivial. The integral curves for the amplitudes are highly nonlinear. The amplitude equations are integrated with some initial conditions, $A_{0}$ and $B_{0}$ and the expression

$$
S=\sum_{i=1}^{N}\left\{\left[A\left(t_{i} ; \lambda\right)-A_{i}\right]^{2}+\left[B\left(t_{i} ; \lambda\right)-B_{i}\right]^{2}\right\}
$$

is minimized with respect to the 10 variational parameters, $\lambda$, namely the 2 growth-rates, the 4 cubic coefficients and the 2 sets of initial conditions, $A(0)$ and $B(0)$. Here $A(t ; \lambda)$ and $B(t ; \lambda)$ comprise the integral curves for both initial conditions, and $A_{i}$ and $B_{i}$ are the amplitudes at points $t_{i}$ for both runs, which are to bê fitted.

The fits are shown in the figures.

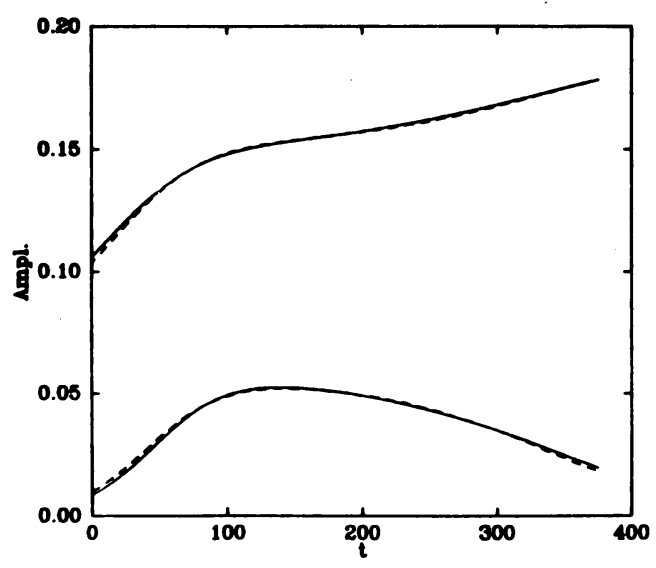

FIG. 1a

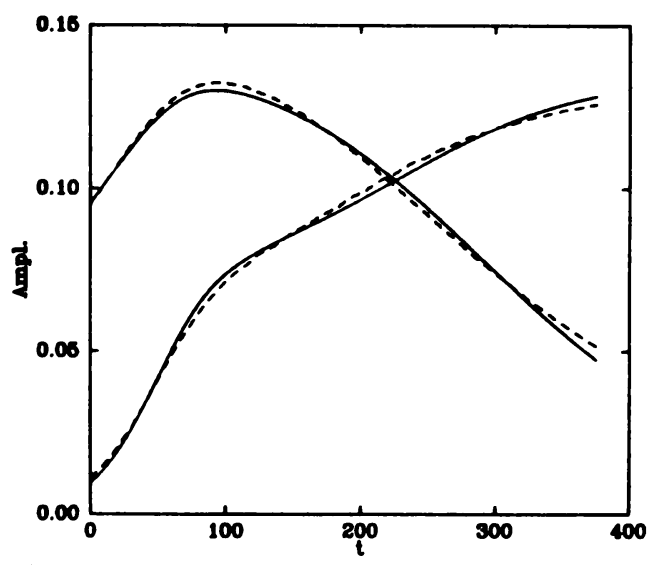

FIG. 2a

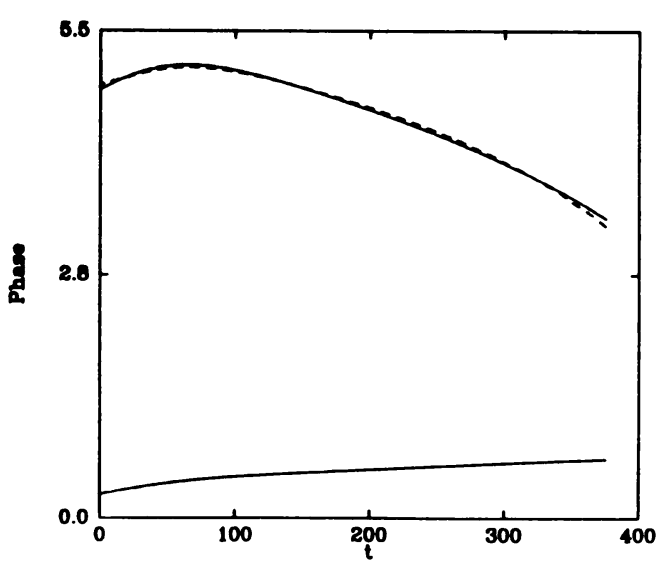

FIG. 1b

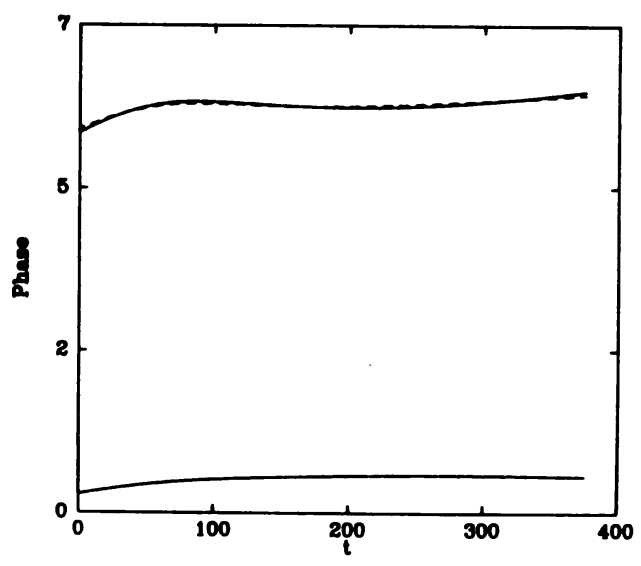

FIG. $2 b$ 
The dashed curves represent the Fourier fits to the hydro-runs and the solid curves the integral curves of the amplitude equations for the 2 different initial conditions, one of which leads to a nonlinear pulsation, which in lowest order corresponds to the fundamental mode (Fig. 1) and the other to the first overtone (Fig. 2). The Figures (a) refer to the amplitudes and (b) to the phases.

In Fig. 3 we exhibit the evolution of the model in an amplitudeamplitude plot, similar to the ones discussed in Buchler and Kovacs (1986b).

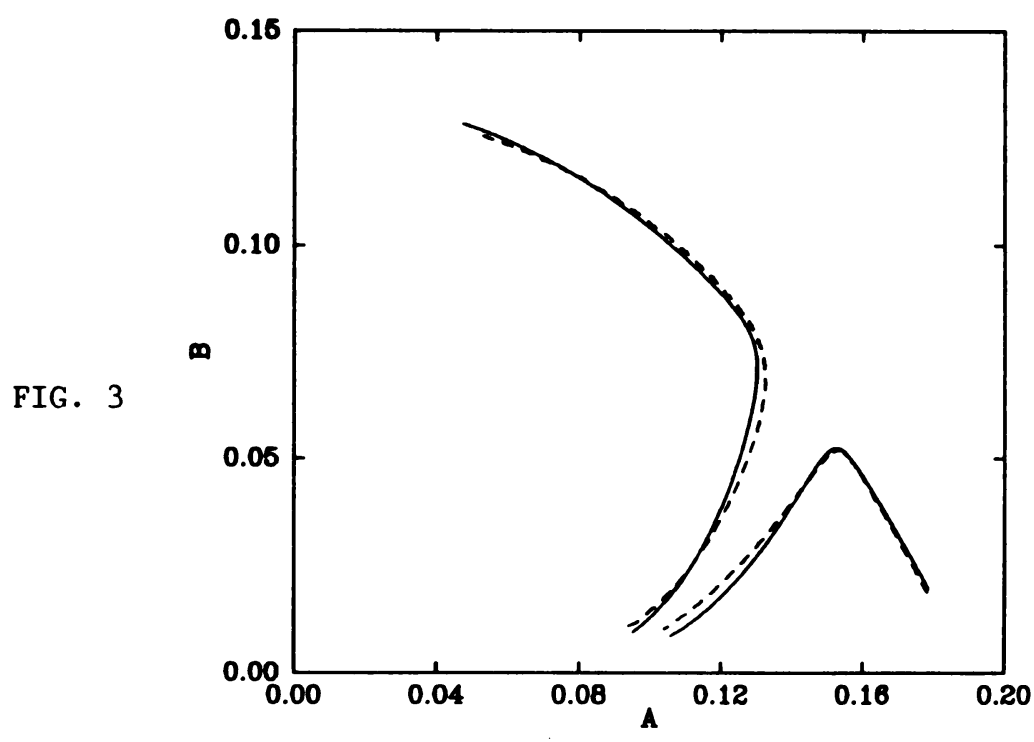

The model parameters, which have been obtained from the fit are shown in the following Table.

\section{integral $\mathrm{fit}$}

$\begin{array}{lll}K_{0}=0.0081 & K_{1}=0.0560 \\ \bar{K}_{0}=-0.0185 & \bar{K}_{1}=-0.0076\end{array}$

$A_{1 c}=0.191 \quad B_{1 c}=0.135$

$\operatorname{Re} Q_{0}=-0.222 \quad \operatorname{Re} \mathrm{T}_{0}=-0.864$

$\operatorname{Re} Q_{1}=-3.076 \quad \operatorname{Re} \mathrm{T}_{1}=-2.040$

Im $Q_{0}=-0.1208$ Im $T_{0}=-0.2791$

$\operatorname{Im} Q_{1}=-0.8429 \quad \operatorname{Im} \mathrm{T}_{1}=-0.8825$

$\bar{v}_{0}=1.84781 \quad \bar{v}_{1}=2.47925$ numerical relaxation method

$$
\begin{array}{ll}
K_{0}=0.0078 & K_{1}=0.0547 \\
\bar{K}_{0}=-0.0185 & \bar{K}_{1}=-0.0050 \\
A_{1 c}=0.191 & B_{1 c}=0.134
\end{array}
$$

The fitted values of the $K$ are in excellent agreement with the 
linear nonadiabatic growth-rates. The cubic coefficients are all negative, as conjectured by Buchler and Kovacs (1986b). Also shown in the table are the nonlinear growth-rates $\bar{K}$ and the limiting amplitudes ( $A_{1}$ and $B_{1 C}$ ), derived from the fit, as well as the $\bar{k}$ (Floquet coefficientsf and limiting amplitudes computed with the numerical hydrodynamic relaxation code of Stellingwerf. It must be considered a great success of the amplitude equation formalism that the limiting behavior of the model and its stability can be predicted from limited and transient numerical hydrodynamic time-integrations.

The fits to the amplitude equations give a clue as to why only the first two modes appear, except for very short-lived transients. The fit gives $\mathrm{T}_{0}<0$ and $\mathrm{T}_{1}<0$ and, reasonably, one also expects $\mathrm{T}_{\nu}<0$ for the other modes. On the other hand, the nonlinear stability coefficients ("mode $v$ stable in the fundamental", e.g.) are given by

$$
\bar{K}_{v}=K_{v}+T_{v 0} A^{2},
$$

so that a linearly stable mode $(K<0)$ becomes even more stable through the nonlinear interaction. A linearly unstable mode, on the other hand, may temporarily get excited until the amplitude of the dominant mode gets sufficiently large and stabilizes it.

Some small discrepancies remain and we list several possible causes: first, the model has some low order resonances, typical of $R R$ Lyrae models, namely $2 \omega_{0} \sim \omega_{3}$ and $2 \omega_{1}-\omega_{0} \sim \omega_{2}$, which we have disregarded, but which are bound to have some, albeit a small, effect; second, the amplitude equations have been truncated at the lowest nonlinearities and the next order, quintic ones may not be totally negligible; finally, the Fourier fit has been limited to 3rd order frequency combinations.

In conclusion, it is remarkable how well the essence of the results of a complicated hydrodynamical integration is captured by just two (complex nonresonant) lowest order amplitude equations for the two dominant modes.

This work has been supported by NSF (AST84-10631).

\section{REFERENCES}

Buchler, J. R. 1985 in Chaos in Astrophysics, Eds. Buchler, J. R. et al., NATO ASI Ser. C 161, 137.

Buchler, J. R. and Goupil, M. J. 1984, Ap. J. 279, 394.

Buchler, J. R. and Kovács, G. 1986a, Ap. J. 303, 749.

Buchler, J. R. and Kovács, G. 1986b, Ap. J. (sep 15)

Coullet, P. and Spiegel, E. A. 1984, SIAM J. Appl. Math, 43, 776.

Klapp, J., Goupil, M. J. and Buchler, J. R. 1985, Ap. J., 296, 514.

Kovács, G. Buchler, J. R. and Davis, C. G. 1986 Ap. J. (submitted)

Spiegel, E. A. 1985 in Chaos in Astrophysics, loc. cit., 91.

Stellingwerf, R. S. 1974, Ap. J. 192, 139. 